\title{
BIBECHANA
}

A Multidisciplinary Journal of Science, Technology and Mathematics

ISSN 2091-0762 (online)

Journal homepage: http://nepjol.info/index.php/BIBECHANA

\section{Almost boundedness and matrix transformation}

\author{
Ab Hamid Ganie \\ Department of Mathematics, \\ National Institute of Technology, Srinagar (India)- 190006. \\ Email: ashamidg@rediffmail.com \\ Article history: Received 25 September, 2012; Accepted 12 November, 2012
}

\begin{abstract}
The sequence space $a_{c}^{r}$ have been defined and the various classes of infinite matrices have been characterized by Aydin and Başar, ( see, [1] ), where $1 \leq p \leq \infty$. In this paper we characterize the classes $\left(a_{c}^{r}: f_{\infty}\right),\left(a_{c}^{r}: f\right)$ and $\left(a_{c}^{r}: f_{0}\right)$, where $f_{\infty}, f$ and $f_{0}$ denote respectively the spaces of almost bounded sequences, almost convergent sequences and almost convergent null sequences.
\end{abstract}

Keywords: Sequence space of non-absolute type; almost convergent sequences; $\beta$-duals and Matrix Transformations.

\section{Introduction, Background and Preliminaries}

A sequence space is defined to be a linear space with real or complex sequences. Throughout the paper $\mathbb{N}, \mathbb{R}$ and $\mathbb{C}$ denotes the set of non-negative integers, the set of real numbers and the set of complex numbers, respectively.

Let $\omega$ denote the space of all sequences ( real or complex ). Let $X$ and $Y$ be two non-empty subsets of $\omega$. Let $A=\left(a_{n k}\right),(n, k \in \mathbb{N})$, be an infinite matrix of real or complex numbers. We write $(A x)_{n}=$ $A_{n}(x)=\sum_{k} a_{n k} x_{k}$. Then $A x=\left\{A_{n}(x)\right\}$ is called the $A$-transform of $x$, whenever $A_{n}(x)=$ $\sum_{k} a_{n k} x_{k}<\infty$ for all $n \in \mathbb{N}$. We write $\lim _{n} A x=\lim _{n} A_{n}(x)$. If $x \in X$ implies $A x \in Y$, we say that $A$-defines a matrix transformations from $X$ into $Y$, denoted by $A: X \rightarrow Y$. By $(X: Y)$, we mean the class of all matrices $A$ such that $A: X \rightarrow Y$.

For a sequence space, the matrix domain $X_{A}$ of an infinite matrix $A$ is defined as

$$
\text { (1) } X_{A}=\left\{x=\left(x_{k}\right) \in \omega: A x \in X\right\}
$$

Let $\ell_{\infty}$ and $c$ be the Banach spaces of bounded and convergent sequences $x=\{x(n)\}_{n=0}^{\infty}$ with supremum norm $\|x\|=\sup _{n}|x(n)|$. Let $T$ denote the shift operator on $\omega$, that is,$T x=\{x(n)\}_{n=1}^{\infty}$, $T^{2} x=\{x(n)\}_{n=2}^{\infty}$ and so on. A Banach limit $L$ is a non-negative linear functional 
on $\ell_{\infty}$ such that $L$ is invariant under the shift operator and $L(e)=1$, where $e=(1,1, \ldots)$ (see, [2]), that is, a functional $L: \ell_{\infty} \rightarrow \mathbb{R}$ is called a Banach limit if

(i) $\quad L$ is linear,

(ii) $\quad L(x) \geq 0$ if $x_{n} \geq 0$ for all $n$.

(iii) $L(x)=L(T x)$ where $T$ is shift operator on $\omega$.

(iv) $\quad L(e)=1$, where $e=(1,1, \ldots)$.

Since the Hahn-Banach norm preserving extension is not unique, there must be many Banach limits in the dual space of $\ell_{\infty}$, and usually different Banach limits have different values at the same element in $\ell_{\infty}$. However, there indeed exists sequences whose values of all Banach limits are same. If $x=$ $\left\{x_{n}\right\}_{n=0}^{\infty} \in c$, where $c$ is a Banach space of $\ell_{\infty}$ consisting of convergent sequences, then $L(x)=\lim _{n} x_{n}$ is a trivial example. Besides this there also exists non-convergent sequences satisfying this property. For example $x=\{1,0,1,0, \ldots\}$ the value of $L(x)=\frac{1}{2}$ is same for every Banach limit. Lorentz (see, [4]) called a sequence $x=\left\{x_{n}\right\}_{n=1}^{\infty}$ almost convergent if all Banach limits of $x, L(x)$, are same, and this unique Banach limit is called $F$-lim of $x$. In his paper Lorentz proved the following criterion for almost convergent sequences.

A $x=\left\{x_{n}\right\}_{n=0}^{\infty} \in \ell_{\infty}$ is almost convergent with $F$-limit $L(x)$ if and only if

$$
\lim _{p \rightarrow \infty} t_{m n}(x)=L(x)
$$

where, $t_{m n}(x)=\frac{1}{p} \sum_{i=0}^{p-1} T^{i} x_{n},\left(T^{0}=0\right)$, uniformly in $n \geq 0$.

The above limit can be rewritten in detail as

$$
(\forall \varepsilon>0),\left(\exists p_{0}\right)\left(\forall p>p_{0}\right)(\forall n)\left|\frac{x_{n}+\cdots+x_{n+p-1}}{p}-L\right|<\varepsilon .
$$

We denote the set of almost convergent sequences by $f$.

$$
f=\left\{x \in l_{\infty}: \lim _{m} t_{m n}(x) \text { exists, uniformly in } n\right\} .
$$

Nanda [6] has defined a new set of sequences $f_{\infty}$ as follows:

$$
f_{\infty}=\left\{x \in l_{\infty}: \lim _{m}\left|t_{m n}(x)\right|<\infty\right\} .
$$

We call $f_{\infty}$ the set of all almost bounded sequences. The approach of constructing a new sequence space by means of matrix domain of a particular limitation method has been studied by several authors viz., ( see, $[1,5,7])$.

Following (see, [1], [7]), the sequence space $a_{c}^{r}$ is defined as the set of all sequences whose $A^{r}$-transform is in $c$, that is,

$$
a_{c}^{r}=\left\{x=\left(x_{k}\right) \in \omega: \lim _{n} \frac{1}{n+1} \sum_{k=0}^{n}\left(1+r^{k}\right) x_{k} \text { exists }\right\}
$$


where, $a_{n k}^{r}=\left\{\begin{array}{cl}\frac{1+r^{k}}{n+1}, & 0 \leq k \leq n, \\ 0, & k>n .\end{array}\right.$

With the notation of (1) that, $a_{c}^{r}=(c)_{A^{r}}$.

\section{Main Results}

Define the sequence $y=\left(y_{k}(r)\right)$ which will be used, by the $A^{r}$-transform of a sequence $x=\left(x_{k}\right)$, that is,

$$
y_{k}(r)=\sum_{j=0}^{k} \frac{1+r^{j}}{k+1} x_{j} ; \text { for } k \in \mathbb{N} .
$$

For brevity in notation, we write

$$
t_{m n}(A x)=\frac{1}{m+1} \sum_{j=0}^{m} A_{n+i}(x)=\sum_{k} a(n, k, m) x_{k}
$$

where, $a(n, k, m)=\frac{1}{m+1} \sum_{j=0}^{m} a_{n+j, k} \quad ;(n, k, m \in \mathbb{N})$

$$
\text { Also }, \quad \tilde{a}(n, k, m)=\Delta\left[\frac{a(n, k, m)}{1+r^{k}}\right](k+1)=\left[\frac{a(n, k, m)}{1+r^{k}}-\frac{a(n, k+1, m)}{1+r^{k+1}}\right](k+1)
$$

We denote by $X^{\beta}$, the $\beta$-deal of a sequence space $X$ and mean the set of all the sequences $x=\left(x_{k}\right)$ such that $x y=\left(x_{k} y_{k}\right) \in c s$ for all $y=\left(y_{k}\right) \in X$. Now, we give the following lemmas which will be needed in proving the main Theorems.

Lemma 2.1[1]: Define the sets $D_{1}(p)$ and $D_{2}(p)$ as follows

$$
\begin{aligned}
& D_{1}^{r}=\left\{a=\left(a_{k}\right) \in \omega: \sum_{k}\left|\Delta\left(\frac{a_{k}}{1+r^{k}}\right)(k+1)\right|<\infty\right\} \\
& D_{2}^{r}=\left\{a=\left(a_{k}\right) \in \omega:\left(\frac{a_{k}}{1+r^{k}}\right) \in c s\right\}
\end{aligned}
$$

where, $\quad \Delta\left(\frac{a_{k}}{1+r^{k}}\right)=\frac{a_{k}}{1+r^{k}}-\frac{a_{k}}{1+r^{k+1}}$

Then, $\quad\left[a_{c}^{r}\right]^{\beta}=D_{1}^{r} \bigcap D_{2}^{r}$

Lemma 2.2 [5]: $f \subset f_{\infty}$

Theorem 2.1: $\quad A \in\left(a_{c}^{r}: f_{\infty}\right)$ if and only if 


$$
\sup _{n, m \in \mathbb{N}} \sum_{k}|\tilde{a}(n, k, m)|<\infty
$$

and

$$
\left\{\frac{a_{n k}}{1+r^{k}}\right\}_{k \in \mathbb{N}} \in c s, \text { for all } n \in \mathbb{N}
$$

Proof: Sufficiency: Suppose the conditions (3) \& (4) holds and $x \in a_{c}^{r}$. Then $\left\{a_{n, k}\right\}_{k \in \mathbb{N}} \in\left[a_{c}^{r}\right]^{\beta}$ for every $n \in \mathbb{N}$, the $A$-transform of $x$ exists. Since $x \in a_{c}^{r}$, by hypothesis, and $a_{c}^{r} \cong c$ (see,[1]), we have $y \in c$. Thus, we can find $K>0$ such that $\sup _{k}\left|y_{k}\right|<K$.

$$
\begin{aligned}
\left|t_{m n}(A x)\right| & =\left|\sum_{k} a(n, k, m) x_{k}\right|=\left|\sum_{k} \tilde{a}(n, k, m) y_{k}\right| \\
\leq & \sum_{k}|\tilde{a}(n, k, m)|\left|y_{k}\right| \leq K \sum_{k}|\tilde{a}(n, k, m)|
\end{aligned}
$$

Taking sup on both sides, we get $A x \in f_{\infty}$ for every $\quad x \in a_{c}^{r}$.

Necessity: Suppose that $A \in\left(a_{c}^{r}: f_{\infty}\right)$. Then $A x$ exists for every $x \in a_{c}^{r}$ and this implies that $\left\{a_{n, k}\right\}_{k \in \mathbb{N}} \in\left[a_{c}^{r}\right]^{\beta}$ for every $n \in \mathbb{N}$, the necessity of (4) is immediate. Now, $\sum_{k} a(n, k, m) x_{k}$ exists for each $\mathrm{m}, \mathrm{n}$ and $x \in a_{c}^{r}$, the sequences $\{a(n, k, m)\}_{k \in \mathbb{N}}$ define the continuous linear functionals $\psi_{m n}(x)$ on $a_{c}^{r}$ by

$$
\psi_{m n}(x)=\sum_{k} a(n, k, m) x_{k} ;(n, k, m \in \mathbb{N}) .
$$

Since $a_{c}^{r}$ and $c$ are norm isomorphic ( see [1], ), it should follow with (2) that $\left\|\psi_{m n}(x)\right\|=\|\tilde{a}(n, k, m)\|$ holds for every $k \in \mathbb{N}$. This implies that the functionals defined by $\psi_{m n}$ on $a_{c}^{r}$ are point wise bounded, so by uniform bounded principle, there exists $M>0$ such that

$$
\left\|\psi_{m n}(x)\right\| \leq M \text { for every } m, n \in \mathbb{N} .
$$

Thus we conclude that

$$
\sup _{m, n}\left|\psi_{m n}(x)\right|=\sup _{m, n}\left|\sum_{k} a(n, k, m) x_{k}\right|=\sup _{m, n}\left|\sum_{k} \tilde{a}(n, k, m) y_{k}\right|<M
$$

This implies that $\sup _{n, m \in \mathbb{N}} \sum_{k}|\tilde{a}(n, k, m)|<\infty$, which shows the necessity of the condition (3) and the proof of (i) is complete.

Theorem 2.2: $A \in\left(a_{c}^{r}: f\right)$ if and only if (3),(4) and

(5) $\quad \lim _{m} \tilde{a}(n, k, m)=\beta_{k}$, uniformly in $n$, and for each $k \in \mathbb{N}$.

(6) $\quad \lim _{m} \sum\left|\tilde{a}(n, k, m)-\beta_{k}\right|=0$, uniformly in $n$. 
Proof: Sufficiency: Suppose that the conditions (3), (4), (5) and (6) hold and $x \in a_{c}^{r}$. Then $A x$ exists and at this stage, we observe with the help of (5) \& (6) that

$$
\sum_{j=0}^{k}\left|\beta_{j}\right|=\sup _{m, n} \sum_{j}|\tilde{a}(n, j, m)|<\infty
$$

holds for every $k$. This gives that $\left(\beta_{k}\right) \in l_{1}$. Since $x \in a_{c}^{r}$ by hypothesis and $a_{c}^{r} \cong c$ ( see,[1]), we have $y \in c$.Therefore, we can easily see that $\left(\beta_{k} y_{k}\right) \in l_{1}$ for each $y \in c$ and also there exists $K>0$ such that $\sup _{k}\left|y_{k}\right|<K$. Now for $\varepsilon>0$, choose a fixed $k_{0} \in \mathbb{N}$, there is some $m_{0} \in \mathbb{N}$ such that

$$
\left|\sum_{k=0}^{k_{0}}\left\langle\tilde{a}(n, k, m)-\beta_{k}\right\rangle y_{k}\right|<\frac{\varepsilon}{2}
$$

for every $m \geq m_{0}$ and $k_{0} \in \mathbb{N}$.

Also by (6), there is some $m_{1} \in \mathbb{N}$, such that

$$
\sum_{k=k_{0}+1}^{\infty}\left|\tilde{a}(n, k, m)-\beta_{k}\right|<\frac{\varepsilon}{2}
$$

for every $m \geq m_{1}$ uniformly in $n$.Thus, we have

$$
\begin{aligned}
\mid \frac{1}{m+1} \sum_{j=0}^{m}(A x)_{n+j} & -\sum_{k} \beta_{k} y_{k}|=| \sum_{k}\left\langle\tilde{a}(n, k, m)-\beta_{k}\right\rangle y_{k} \mid \\
& \leq\left|\sum_{k=0}^{k_{0}}\left\langle\tilde{a}(n, k, m)-\beta_{k}\right\rangle y_{k}\right|+\sum_{k=k_{0}+1}^{\infty}\left|\left\langle\tilde{a}(n, k, m)-\beta_{k}\right\rangle y_{k}\right| \\
& <\frac{\varepsilon}{2}+\sum_{k=k_{0}+1}^{\infty}\left|\left\langle\tilde{a}(n, k, m)-\beta_{k}\right\rangle\right|\left|y_{k}\right| \\
& <\frac{\varepsilon}{2}+K \frac{\varepsilon}{2 K}=\varepsilon
\end{aligned}
$$

for all sufficiently large $m$, uniformly in $n$. Hence, $A x \in f$, which proves sufficiency.

Necessity: Suppose that $A \in\left(a_{c}^{r}: f\right)$. Then, since $f \subset f_{\infty}$ ( by Lemma 2.2), the necessities of (3) and (4) are immediately obtained from Theorem 2.1. To prove the necessity of (5), consider the sequence $b^{(k)}(r)=\left(b_{n}^{(k)}(r)\right)$ for every $k \in \mathbb{N}$, where

$$
b_{n}^{(k)}(r)=\left\{\begin{array}{cl}
(-1)^{n-k} \frac{1+k}{1+r^{k}} & , \quad k \leq n \leq k+1 \\
0 & , 0 \leq n<k \text { or } n>k+1
\end{array}\right.
$$

Since $A x$ exists and is in $f$ for each $x \in a_{c}^{r}$, one can easily see that 


$$
A b^{(k)}(r)=\left\{\Delta\left(\frac{a_{n k}}{1+r^{k}}\right)(k+1)\right\}_{n \in \mathbb{N}} \in f \text { for all } k \in \mathbb{N} \text {, which proves the necessity of (6). Similarly }
$$

taking $x=e \in a_{c}^{r}$, we shall get

$$
A x=\left\{\sum_{k} \Delta\left(\frac{a_{n k}}{1+r^{k}}\right)(k+1)\right\}_{n \in \mathbb{N}} \in f, \text { which proves the necessity of (5).This concludes }
$$

the proof.

Note that if we replace $f$ by $f_{0}$, then Theorem 2.2 is reduced to the following corollary:

Corollary: $A \in\left(a_{c}^{r}: f_{0}\right)$ if and only if (3),(4), (5) and (6) holds with $\beta_{k}=0$ for each $k \in \mathbb{N}$.

\section{References}

[1] C. Aydinand F. Başar, On the new sequence space of which include the spaces $c_{0}$ and $c$, Hokkaido Math. J., 33(2) (2004) 83-398.

[2] S. Banach, Theỏries des operations linẻaries, Warszawa, (1932).

[3] C. G. Lascarides and I. J. Maddox, Matrix transformations between some classes of sequences, Proc. Camb. Phil. Soc., 68 (1970) 99-104.

[4] G. G. Lorentz, A contribution to the theory of divergent series, Acta Math., 80(1948)167-190.

[5] Mursaleen, Infinite matrices and almost convergent sequences, Southeast Asian Bulletin of Math. 19(1995) 45-48.

[6] S. Nanda, Matrix transformations and almost boundedness, Glasnik Mat., 14(1979) 99-107.

[7] N. A. Sheikh, and A. H. Ganie, On the $\lambda$-convergent sequence and almost convergence ( to be appeared in Thai J. of Math, vol.3 (2012). 